\section{Oxford Nanopore bests PacBio}

In a patent battle between the two main players in long-read sequencing, the US Court of Appeals for the Federal Circuit in February upheld an earlier ruling that DNA sequencers imported by UK-based Oxford Nanopore Technologies for sale in the United States do not violate two of Pacific Biosciences patents. The dispute came about after PacBio alleged that Oxford Nanopore's MinION and PromethION sequencers infringed one of its patents by using an identical method to its own invention to analyze DNA strands. Later, PacBio amended its filing to allege that Oxford Nanopore's products infringed a second patent. The recent one-sentence decision by the Court of Appeals affirms the February 2018 ruling by the International Trade Commission (ITC) in favor of Oxford Nanopore, but does not expand on its reasons; however, the ruling comes on the heels of a European Patent Office decision in January revoking PacBio's patent EP3045542, with claims to a single molecule sequencing process, which was challenged by Oxford Nanopore. "The ITC correctly held that Oxford Nanopore's technology does not infringe PacBio's patents," Stephen Hash, the attorney representing Oxford Nanopore, told legal publication Law360. "We are very happy with the Federal Circuit's affirmance of that determination." Gordon Sanghera, Oxford Nanopore's $\mathrm{CEO}$, added in a statement, "Pacific Biosciences has not succeeded in its strategy to try to prevent us from selling our real-time, direct sequencing technology."

Published online: 2 April 2019 https://doi.org/10.1038/s41587-019-0098-y

\footnotetext{
“Making a documentary about Crispr must feel like building a rocket ship while it's warping through a wormhole." @MeganMolteni. Several CRISPR documentaries are in the works. (Twitter, 6 March 2019)
}

"Small teams remember forgotten ideas, ask questions and create new directions, whereas large teams chase hotspots and forget less popular ideas, answer questions and stabilize established paradigms." Lingfei Wu. Analysis of 65 million documents showed that small teams are more likely to push the envelope than large teams. (Science Daily, 13 February 2019)

“Martin Shkreli's prison to-do list: 15 push-ups in a row, and running his former pharmaceutical company by contraband smartphone."

The disgraced pharmaceutical executive plans a billion-dollar comeback from his prison cell. (The Wall Street Journal, 8 March 2019) in a trade-off. "There isn't a single microdystrophin that's perfect," says DMD pioneer Kay Davies of Oxford University. "There are shortcomings with all of them, and each involves a compromise."

The three DMD gene therapies in the clinic are also distinguished by the AAV vector they employ and the particular muscle-specific promoter that drives microdystrophin expression. Both Solid's and Pfizer's agents are based on AAV serotype 9 , selected for its tropism for skeletal muscles and the diaphragm; Sarepta's gene therapy uses AAVrh74, an AAV isolated from rhesus macaques that has a tropism for muscle. This virus is also expected to elicit less pre-existing immunity than human AAVs, to which patients may have been previously exposed. The companies also employ different promoters. Which gene, vector and promoter combination works best will become clearer as trials progress.

The goal for any DMD gene therapy is to achieve at least $10 \%$ of the dystrophin abundance in muscle seen in people without DMD mutations; this is where Solid's SGT-001 fell short. Even so, "all patients showed microdystrophin," says Jorge Quiroz, chief medical officer at Solid. In one patient microdystrophin was detectable in roughly $10 \%$ of muscle fibers, which, says Quiroz, is comparable to levels seen in Becker muscular dystrophy. But in the other two patients, microdystrophin expression was so low as to be undetectable. "Obviously, we were aiming for more," he admits.

These disappointing results contrast with those reported by Sarepta, which says that its agent achieved expression of microdystrophin in $>80 \%$ of muscle fibers and at $74-96 \%$ of normal protein abundance in a phase $1 / 2$ trial. Pfizer, which initiated a phase $1 \mathrm{~b}$ dose-escalation trial of its DMD gene therapy DMD PF-06939926 in March 2018 after receiving orphan drug status in both the USA and Europe in May 2017, has yet to report comparable data. A company spokesperson did, however, confirm that six boys have been treated, three at the initial study dose and three at the next higher dose. Pfizer plans to share early data from the trial in the first half of this year.

Although investors delivered a harsh verdict on SGT-001, with Solid's shares dropping $70 \%$ after the news, the company remains upbeat. The IGNITE trial is a single ascending-dose study, and the recent report was based on three patients who received the lowest dose in the protocol. "The logical next step is to dose escalate, and that's what we're planning to do," says Quiroz.
Whether Solid and Sarepta do better may hinge not just on the therapies' designs but also on trial protocols, such as the age of patients and their stage of disease. The first patient Solid treated was an adolescent, whereas Sarepta's patients have all been younger than seven. "If you go in too early, muscle growth could throw the virus out, but if you go in too late there isn't enough muscle left," says Davies. "The therapeutic window might be quite narrow, though this hasn't been defined yet." In addition, the patients in these trials have also had different histories of exposure to corticosteroids. Although this is unlikely to affect the gene therapy's function, it could influence how well the immune system may tolerate the AAV vectors and other clinical outcomes.

Exon skipping takes a different approach to correcting dystrophin. Rather than supplement the defective gene, exon skipping uses ASOs to direct the cell's splicing machinery to ignore the mutated exon and the out-of-frame exons that lie downstream, to enable the reading to restart at the correct reading frame. As deletions can occur in many different exons, the therapies are specific for skipping the affected exons, and although any particular exon-skipping therapy is only be applicable in $15 \%$ or fewer of DMD cases, together they cover about $80 \%$ of DMD patients.

The first approval for an exon-skipping drug was, however, controversial. Sarepta's Exondys was approved on the basis of inconclusive results from a 12-patient trial. The agent is designed to treat patients with genetic mutations in exon 51. In September 2016, the biotech received accelerated approval from the FDA, with continued approval being contingent on further evidence of clinical benefit. On 14 February, the FDA accepted a New Drug Application for another of Sarepta's exon skipping therapies, the exon-53-skipping therapy golodirsen (SRP-4053); Sarepta also has SRP-4045, targeting exon 45, in a phase 3 trial, and other ASOs in preclinical and discovery programs for DMD. Another company, Nippon Shinyaku, headquartered in Kyoto, Japan, reported increased dystrophin expression in 14 out of 16 boys aged 5-12 years of age in a phase $1 / 2$ trial of its exon-53-skipping phosphorodiamidate morpholino ASO viltolarsen in October 2018.

Wave Life Sciences is also pursuing exon-skipping DMD therapies based on its proprietary phosphorothioate stereopure chemistry. Wave says this produces a single nucleic acid isomer instead of a mix of hundreds of thousands 